TITTLE PAGE

\title{
Across-subject calibration of an instrumented glove to measure hand movement for clinical purposes
}

\author{
Author Names \\ Verónica Gracia-Ibáñez ${ }^{1}$, Margarita Vergara ${ }^{1}$, James H. Buffi ${ }^{2,3}$, Wendy M. Murray ${ }^{2,3,4}$, \\ Joaquín L. Sancho-Bru ${ }^{1}$ \\ Filiation \\ ${ }^{1}$ Department of Mechanical Engineering and Construction, Universitat Jaume I, Castelló, \\ Spain; \\ ${ }^{2}$ Department of Biomedical Engineering, Physical Medicine and Rehabilitation, and \\ Physical Therapy and Human Movement Sciences, Northwestern University, Chicago, IL, \\ USA; \\ ${ }^{3}$ Sensory Motor Performance Program, Rehabilitation Institute of Chicago, Chicago, IL, \\ USA; \\ ${ }^{4}$ Research Service, Edward Hines, Jr. VA Hospital, Hines, IL, USA
}

Corresponding author:

\section{Verónica Gracia-Ibáñez}

Department of Mechanical Engineering and Construction

Universitat Jaume I

Avinguda Vicent Sos Baynat, s/n.

Tel. +34 964728915

Fax +34964728106

Castelló 12071, Spain

e-mail: vgracia@uji.es

\section{Funding:}

This work was supported by the Spanish Ministry of Economy and Competitiveness under Grant [project DPI2014-52095-P]; Universitat Jaume I under Grant [projects P1-1B201333 and P1- 1B2014-10] and also by the NIH Grant NIH R01EB011615. 


\title{
Across-subject calibration of an instrumented glove to measure hand movement for clinical purposes
}

\begin{abstract}
Motion capture of all degrees of freedom of the hand collected during performance of daily living activities remains challenging. Instrumented gloves are an attractive option because of their higher ease of use. However, subject-specific calibration of gloves is lengthy and has limitations for individuals with disabilities. Here, a calibration procedure is presented, consisting in the recording of just a simple hand position so as to allow capture of the kinematics of 16 hand joints during daily life activities even in case of severe injured hands. 'across-subject gains' were obtained by averaging the gains obtained from a detailed subject-specific calibration involving 44 registrations that was repeated three times on multiple days to 6 subjects. In additional 4 subjects, joint angles that resulted from applying the 'across-subject calibration' or the subject-specific calibration were compared. Global errors associated with the 'across-subject calibration' relative to the detailed, subjectspecific protocol were small (bias: $0.49^{\circ}$; precision: $4.45^{\circ}$ ) and comparable to those that resulted from repeating the detailed protocol with the same subject on multiple days $\left(0.36^{\circ} ; 3.50^{\circ}\right)$. Furthermore, in one subject, performance of the 'across-subject calibration' was directly compared to another fast calibration method, expressed relative to a videogrammetric protocol as a gold-standard, yielding better results.
\end{abstract}

Keywords: Instrumented glove, across-subject calibration, fast calibration, hand movement, hand disabilities.

\section{INTRODUCTION}

The ability of the human hand to grasp and manipulate objects is a key factor determining an individual's ability to complete a great number of activities of daily living (ADL) as well as of working life (Bullock et al. 2013; Vergara et al. 2014; Zheng, De La Rosa, and Dollar 2011). The versatility of the human hand is possible thanks to the complex 
kinematics of the system: 25 degrees of freedom (DoFs) controlled by muscles, tendons and ligaments (Brand and Hollister 1999). Measurement of complex hand movements is useful for numerous applications, including functional assessment of the pathological hand and its rehabilitation (Chiu et al. 2000; Nathan, Johnson, and McGuire 2009; Oess, Wanek, and Curt 2012), analysis of sporting techniques and ergonomics of tools, the study of human motor control strategies, and robotics (Griffin et al. 2000; Grinyagin, Biryukova, and Maier 2005; Sanchez-Margallo et al. 2010; Tripp et al. 2006).

Different methods can be used to measure hand movement, but most of them fail when applied to the simultaneous measurement of all hand DoFs while performing functional ADL. Goniometers do not allow for the simultaneous measurement of all DoFs. Electromagnetic systems (Mitobe et al. 2006) are susceptible to magnetic and electrical interference from metallic objects in the environment. Marker-based optical systems provide high accuracy (Joaquin L Sancho-Bru et al. 2014), but they can be used only within the area covered by the cameras, require a substantial amount of time to setup the markers, and markers often become occluded during the recording of tasks. Markerless optical motion capture (Metcalf et al. 2013) and inertial systems (Kortier et al. 2014) are frequently adopted in virtual reality games, but even though great enhancements in accuracy are being done ( $\mathrm{O}$ 'flynn et al. 2015), no commercial devices are currently available according to our knowledge. At this point, instrumented gloves seem to be the most effective method for collecting data from all finger joints continuously, without occluding problems, and with no special environmental constraints (J. Buffi et al. 2014).

Despite the relative strengths described above, the use of instrumented gloves is also problematic, primarily due to difficulties associated with the calibration processes needed 
to obtain the gains for the individual sensors that record each DoF. On the one hand, gloves include a high number of sensors to be calibrated. Furthermore, although each individual sensor is linear, some of them do not have only a linear relationship with the anatomical angle to be measured (Eccarius, Bour, and Scheidt 2012), because they are affected by other movements of the same joint (e.g. the abduction sensors at metacarpophalangeal joints, affected by the relative flexion between adjacent joints) or because of their placement they do not measure directly the anatomical angle (e.g. abduction and roll of the thumb). In order to correct this effect, a subject-specific calibration obtained by positioning the fingers in specific angles of combined flexion/extension and abduction spanning the entire range of motion has been shown to provide good accuracy (Eccarius, Bour, and Scheidt 2012). However, this method requires subjects to pose in a large number of postures, along with recording controlled movements, limiting its feasibility for use in real, clinical applications and large-scale field studies. This issue is especially problematic when dealing with patients with disabilities that interfere with the capacity to achieve postures needed for the calibration. In contrast, optimization methods have been used in an attempt to minimize the number of postures/movements required for the calibration (Griffin et al. 2000): each finger and the thumb are repeatedly flexed and extended while maintaining digit tip contact (close loop method), and the gains are optimized such that the joint angles obtained from an underlying model best maintained digit tip contact throughout the task. However, when evaluated against a gold-standard, low accuracy was observed (J. Buffi et al. 2014). In a third approach, artificial neural networks (ANN) have been used to estimate sensor gains from an individual subject's hand segment lengths (Zhou, Malric, and Shirmohammadi 2010). However, this approach requires a large number of previously 
performed manual calibrations on many subjects, spanning a broad range of different segment lengths. In addition, as no angular errors were reported, it is unclear whether the lengths of hand segments are enough to yield high quality data (Zhou, Malric, and Shirmohammadi 2010).

In brief, subject-specific instrumented glove calibration procedures are lengthy and not applicable to patients with some disabilities. In this work we test whether an 'across-subject calibration', defined via detailed, accurate, yet lengthy calibration from a small number of subjects, yields valid data when applied to additional subjects via registration of a single, simple reference posture.

\section{METHODS}

The experiment, approved by the University's Ethical Committee, was developed in three phases. First, a very detailed calibration protocol was applied several times to 6 subjects. The gains obtained through this detailed calibration process were then used to define a 'across-subject calibration'. In a separate group of an additional 4 subjects, the joint angles that resulted from applying this 'across-subject calibration' were compared to those that resulted from transforming the identical set of sensor outputs to joint angles via the detailed, subject-specific calibration method. Finally, in one subject, the errors associated with the across-subject calibration were directly compared to those from another fast calibration method (J. Buffi et al. 2014). In this case, errors were expressed relative to a previously validated videogrammetric protocol (Joaquin L Sancho-Bru et al. 2014). All the errors and comparisons were made on the calculated angles of five different static postures. All the participants were right-handed, free of hand lesions or pathologies and gave 
informed consent to participate. The instrumented glove used was a right-hand Cyberglove (Cyberglove Systems LLC; San Jose, CA), one sized, with 18 resistive flex sensors and 8bit digital signal output proportional to the underlying bending angle (Figure 1), technically described with more detail elsewhere (Eccarius, Bour, and Scheidt 2012). Only outputs from 16 sensors were used in this experiment, discarding the two sensors of the wrist.

\section{Insert Figure 1 here}

\section{Calibration Protocols}

\section{Detailed calibration protocol}

Our protocol combines procedures based on several previous works (J. H. Buffi, Crisco, and Murray 2013; Eccarius, Bour, and Scheidt 2012; Griffin et al. 2000), and consists in registering 44 different poses or guided movements (Figure 2). The first 20 calibration trials correspond to the calibration of 10 individual flexion sensors (two static postures per sensor, F1 to F10, see Fig. 1) to measure flexion at all metacarpophalangeal (MCP1 to MCP5; 1 to 5, thumb to little digit, respectively) and interphalangeal (IP1 and PIP2 to PIP5) joints (Figure 2.a). Gains of these flexion sensors $\left(\mathrm{G}_{\mathrm{F}}\right)$ assume a linear relationship between the flexion angle at these joints and the glove output signals (Eccarius, Bour, and Scheidt 2012; Kessler, Hodges, and Walker 1995; Zhou, Malric, and Shirmohammadi 2010). Each MCP joint of the fingers and all IP joints were calibrated at $0^{\circ}$ and $75^{\circ}$ by pressing custom-made wood tools against the dorsal aspect of the digit. MCP1 was calibrated at $0^{\circ}$ and $35^{\circ}$.

Insert Figure 2 here 
Trials 21 and 22 (Figure 2.b) were used to obtain the gains of A2 to A4 sensors $\left(G_{A}\right)$, corresponding to relative abduction of $\mathrm{MCP} 2$ to $\mathrm{MCP} 5$, also assuming a linear relationship. Both trials correspond to static postures with the hand resting flat on a table, the first with the fingers close together, defined to be $0^{\circ}$ for the three abduction angles, and the second with custom-made wedge tools inserted firmly between the fingers that constrained the relative abduction angles to $25^{\circ}, 16^{\circ}$ and $17^{\circ}$ for MCP2, MCP4 and MCP5, respectively.

Previous studies have warned about the cross-coupling effect between abduction and flexion MCP angles: due to the physical configuration of the glove, the output signal of abduction sensors varies when the adjacent MCP joints flex, even with no variation of the abduction angle, so that the abduction angle needs a correction (Eccarius, Bour, and Scheidt 2012; Zhou, Malric, and Shirmohammadi 2010). We confirmed that a second order polynomial of the flexion angles of adjacent MCP joints provides a good correction for abduction angles, in accordance with Eccarius et al. (Eccarius, Bour, and Scheidt 2012). The 5 polynomial coefficients of the correction term $\left(\mathrm{C}_{1}, \mathrm{C}_{2}, \mathrm{C}_{3}, \mathrm{C}_{4}\right.$ and $\left.\mathrm{C}_{5}\right)$ at each sensor were obtained through an optimization process, by minimizing the root mean square error (RMSE) of the abduction angles measured during six motions with $0^{\circ}$ of abduction. In the case of index-middle abduction, the subject performed three extension-flexion cycles of the index finger with no abduction, while the others three fingers were kept fixed at different MCP flexion angles: $0^{\circ}, 40^{\circ}$ and $80^{\circ}$, (Figure 2.c, trials 23 to 25 ) and then three extensionflexion cycles of the middle, ring and little fingers together, while the index finger was fixed at the same three $\mathrm{MCP}$ angles $\left(0^{\circ}, 40^{\circ}\right.$ and $\left.80^{\circ}\right)$, with no abduction (trials 26 to 28 ). Analogous corrections have been considered for the abduction of MCP4 and MCP5, through trials 29 to 34, and 35 to 40, respectively (Figure 2.d). 
The positions of A1 and Roll1 sensors do not correspond exactly to either flexion or abduction of the thumb carpometacarpal (CMC1) joint (Kramer 1996), making obtaining these joint angles difficult (Crasborn et al. 2006). For CMC1 flexion, we have considered a linear relationship (gain $\mathrm{G}_{\mathrm{F}}$ ) with Roll1 plus an adjustment factor $\left(\mathrm{AF}_{\mathrm{F}}\right)$ with $\mathrm{A} 1$ sensor. The adjustment factor was obtained by minimizing the RMSE of the abduction angles, assumed to be zero, in trial 41, which consists in extending the thumb from neutral (Figure 2.e) to maximal extension (Figure 2.f), and returning to neutral. Analogously, for $\mathrm{CMC1}$ abduction, a linear relationship (gain $\left.\mathrm{G}_{\mathrm{A}}\right)$ with $\mathrm{A} 1$ plus an adjustment factor $\left(\mathrm{AF}_{\mathrm{A}}\right)$ with Roll1 sensor was considered. The adjustment factor was obtained by minimizing the RMSE of the flexion angles, assumed to be zero, in trial 42 , which consists in abducting the thumb from neutral (Figure 2.e) to maximal abduction, then to the maximal adduction (Figure 2.g), and returning to neutral abduction. Once the adjustment factors were calculated, the gains for both linear relationships $\left(\mathrm{G}_{\mathrm{F}}\right.$ and $\left.\mathrm{G}_{\mathrm{A}}\right)$ were obtained from trial 43 , which consists in three consecutive closed loop motions made between index finger and the thumb, repeatedly flexing and extending both digits while maintaining tip contact (Figure 2.h). The gains were calculated so that the joint angles obtained from the underlying kinematic model (Joaquín L. Sancho-Bru et al. 2012) best maintained digit tip contact throughout the task. Index distal interphalangeal (DIP2) flexion angle, not provided by the Cyberglove used in this work, is required for computing the distance between the thumb and index finger tips. This angle was estimated from the PIP2 angle by using the linear regression experimentally obtained with the videogrammetric technique (Joaquin L Sancho-Bru et al. 2014) over 8 subjects performing the same loop movements $\left(D I P 2=0.87 \cdot P I P 2-25.27^{\circ}\right)$. 
Finally, palmar arch is estimated from Roll2 assuming a linear relationship (gain $\mathrm{G}_{\mathrm{F}}$ ) with two postures: previous trial number 21 (palm extended, $0^{\circ}$ ) and trial 44 (palm flexed). In this case, the angle between index-middle knuckles and ring-little knuckles was measured for each subject using a manual goniometer (Figure 2.i).

\section{Fast calibration protocol}

This protocol, based on a previous one (J. Buffi et al. 2014), consists in registering 12 different poses or guided movements. Four trials consist in closed loop motions made between index, middle, ring and little fingers and the thumb, respectively, repeatedly flexing and extending both digits while maintaining tip contact; and they were used to adjust gains of all flexion angles (all $\mathrm{G}_{\mathrm{F}}$ ) and the abduction of thumb CMC together with both adjustment factors (AF) so that the joint angles obtained from the underlying kinematic model (Joaquín L. Sancho-Bru et al. 2012) best maintained digit tip contact throughout the tasks. Again, DIP flexion angles were estimated from the fingers PIP angles by using linear regressions experimentally obtained as with DIP2 $(D I P 3=0.79 \cdot$ PIP3 $\left.18.33^{\circ} ; D I P 4=0.73 \cdot P I P 4-20.54^{\circ} ; \quad D I P 5=0.84 \cdot P I P 5-12.42^{\circ}\right)$.

For abduction of MCP joints of fingers the same procedure as in the detailed protocol was applied to obtain $\mathrm{G}_{\mathrm{A}}$, using analogous postures to trials 21 and 22; but a shortened protocol was applied for the cross-coupling effect, as only the extension-flexion cycles corresponding to $0^{\circ}$ in $\mathrm{MCP}$ flexion of the fixed digits (two trials per sensor) were used.

Across-subject calibration protocol 
The across-subject protocol involved calculating 'across-subject gains' by averaging the gains and coefficients that resulted from the Detailed calibration protocol implemented in a group of 6 subjects (see below, Experimental procedure and analysis, Phase 1).

\section{Joint angle calculation}

Joint angles were calculated using sensor outputs relative to the outputs of trial 21 , which was defined as $0^{\circ}$ for all joints. If $S_{\text {sensor }}$ is the relative output signal of sensor, then the angles at the different joints are calculated as follows.

IP1, PIP2 to PIP5, and MCP1 $1_{\mathrm{f}}$ to $\mathrm{MCP}_{\mathrm{f}}$, angles:

Flexion Angle $=G_{F} \cdot S_{\text {sensor }}$

Palmar arch angle:

Palmar arch Angle= GPalmar $\cdot$ Roll2

MCP2 to MCP5 abduction angles:

$$
\begin{aligned}
M C P 2_{A}= & G_{A 2} \cdot S_{A 2}+\left(C_{1_{M C P 2}} \cdot S_{F 3}+C_{2_{M C P 2}} \cdot S_{F 5}+C_{3_{M C P 2}} \cdot S_{F 3}{ }^{2}+\right. \\
& \left.C_{4_{M C P 2}} \cdot S_{F 5}{ }^{2}+C_{5_{M C P 2}} \cdot S_{F 3} \cdot S_{F 5}\right) \\
\mathrm{MCP}_{A}= & 0 \\
M C P 4_{A}= & G_{A 3} \cdot S_{A 3}+\left(C_{1_{M C P 4}} \cdot S_{F 5}+C_{2_{M C P 4}} \cdot S_{F 7}+C_{3_{M C P 4}} \cdot S_{F 5}{ }^{2}+\right. \\
& \left.C_{4_{M C P 4}} \cdot S_{F 7}{ }^{2}+C_{5_{M C P 4}} \cdot S_{F 5} \cdot S_{F 7}\right) \\
M C P 5_{A}= & M C P 4_{A}+G_{A 4} \cdot S_{A 4}+\left(C_{1_{M C P 5}} \cdot S_{F 7}+C_{2_{M C P 5}} \cdot S_{F 9}+\right. \\
& \left.C_{3_{M C P 5}} \cdot S_{F 7}{ }^{2}+C_{4_{M C P 5}} \cdot S_{F 9}{ }^{2}+C_{5_{M C P 5}} \cdot S_{F 7} \cdot S_{F 9}\right)
\end{aligned}
$$

Thumb CMC flexion and abduction angles:

$$
\begin{aligned}
& C M C 1_{F}=G_{F_{C M C 1}} \cdot\left(\operatorname{Roll1}+A F_{F} \cdot A 1\right) \\
& C M C_{1_{A}}=G_{A_{C M C 1}} \cdot\left(A 1+A F_{A} \cdot \operatorname{Roll} 1\right)
\end{aligned}
$$




\section{Experimental procedure and analysis}

Phase 1. The Detailed calibration was applied to 6 subjects, selected to achieve a representative variation in hand size (Table 1, Sample 1, Subjects 1 through 6). After calibration, sensor outputs were recorded while each subject adopted five static postures (Figure 3), selected to represent different postures incorporating both flexion and abduction of fingers. Each subject repeated the entire process, including calibration and static postures, in three different sessions. The gains and coefficients were calculated from the detailed calibration protocol for each subject and session. The joint angles of the five static postures were estimated from the sensor outputs collected during a given session three times: first, using the gains and coefficients from the corresponding calibration (same session in which the posture was measured); then, from the distinct calibrations resulting from the other two repeated sessions. The differences in the angles that result from transforming the same sensor output with gains from the three repeated calibrations serves as an estimate of the error of using a subject-specific, detailed calibration obtained in a different experimental session and has implications for the need to replicate the calibration for a given subject if testing involves multiple sessions. Mean and standard deviation (SD) across postures and subjects of the paired differences were used as bias and precision errors, respectively. Errors were also evaluated by grouping some of the hand joint movements for a broader interpretation: flexion at all MCP joints, flexion at all IP joints, and abduction at all MCP joints.

After completion of all testing (6 subjects x 3 sessions) and analysis in Phase 1, the 'acrosssubject gains' for the across-subject calibration protocol were defined as the mean values of all the gains and coefficients. 
Insert Table I here

Insert Figure 3 here

Phase 2. Four additional subjects (Table 1, Sample 2, Subjects 7 through 10) were tested in a single session, using the detailed calibration protocol and the same five static postures. The joint angles for each posture were calculated from the sensor output two times in Phase 2: first, using the gains and coefficients resulting from the detailed calibration performed for the subject in the testing session; then, from the across-subject calibration that resulted from Phase 1. Differences between these angles (across-subject minus detailed) provide an estimate of the error of using the 'across-subject calibration' protocol compared to using a detailed subject-specific protocol. Again, mean and SD across postures and subjects of the differences were considered as bias and precision errors. Errors were also evaluated across the grouped hand movements described in Phase 1.

To evaluate the dependence of errors associated with the across-subject calibration on hand-size, Pearson correlations of the precision errors with hand breadth $(\mathrm{HB})$ and hand length (HL) were calculated for each joint angle. The global postures were also visualized using a kinematic hand model developed in previous work (J. H. Buffi, Crisco, and Murray 2013; Holzbaur, Murray, and Delp 2005) comparing both calibrations for each posture and subject.

Phase 3. For a single subject (Subject 9), the errors resulting from the across-subject calibration and the fast calibration protocol were calculated relative to a reference data set, quantified in a separate protocol, using a videogrammetric technique thoroughly detailed ina previous work (Joaquin L Sancho-Bru et al. 2014). Because the hand could not be 
effectively instrumented with the markers needed for the videogrammetric method while simultaneously wearing the instrumented glove, two datasets were collected; the first dataset was collected while the subject was wearing the glove, the second dataset was collected without the glove. Each data set consisted of three trials of each of the five static postures described previously. For the across-subject and fast calibration protocols, joint angles were estimated from the first dataset, using the identical sensor output to calculate the joint angles according to the gains resulting from the respective calibration method. In all comparisons, the average joint postures across all three repetitions for a given posture were compared. The accuracies (bias and precision errors) of the across-subject and fast protocols were calculated as the differences between the angles measured using the respective calibration and the videogrammetric dataset. Notice that the ' $R$ ' American Sign Language (ASL) posture (Figure 3.e) was not included, as it couldn't be measured with the videogrammetric technique because of markers and fingers overlapping.

Phase 4. Finally the clinical utility of the across-subject calibration was tested on a subject with a severely injured hand (dominant hand) caused by an accident with a circular saw, in an advanced recovering stage (Figure 4). The protocol was used to measure the active range of motion (AROM) of his hand joints. His AROM values were compared to the normal values measured with the same protocol to a sample of 24 healthy subjects.

\section{Insert Figure 4 here}

\section{RESULTS}

Phase 1. Global bias and precision errors associated with transforming the same sensor output with gains obtained from three, distinct, detailed subject-specific calibrations 
obtained in repeated sessions are small $\left(0.36^{\circ}\right.$ and $3.50^{\circ}$, respectively; Table 2$)$. Highest bias $\left(3.19^{\circ}\right)$ corresponded to abduction of little MCP joint, and highest precision error $\left(12.66^{\circ}\right)$ to flexion of thumb CMC joint, followed by palmar arch. Very low precision error is observed for IP and MCP flexion angles (2.71 and $1.62^{\circ}$, respectively), and slightly higher for MCP abduction angles $\left(3.52^{\circ}\right)$.

Insert Table II here

Phase 2. Global bias and precision errors associated with using the 'across-subject calibration' protocol compared to using a detailed subject-specific protocol are similar to those obtained in Phase $1\left(0.49^{\circ}\right.$ and $4.45^{\circ}$, respectively; Table 2). Again, highest bias corresponded to abduction of little MCP joint $\left(4.86^{\circ}\right)$, highest precision errors to $\mathrm{CMC}$ joint angles and abduction of little MCP joint (about $10^{\circ}$ ). Precision errors for IP and MCP flexion angles are very small $\left(1.70^{\circ}\right.$ and $2.67^{\circ}$, respectively). Precision errors associated with abduction of MCP joints are somewhat larger. The differences for the worst case are graphically visualized in Figure 5.

Insert Figure 5 here

Joint angle errors were significantly correlated with hand size, especially for PIP and MCP flexion angles (cf., Fig. 6, shaded cells indicate significant Pearson correlations). In general, we observed stronger correlations with measures of hand length compared to breadth.

\section{Insert Figure 6 here}

Phase 3. When the across-subject calibration protocol developed here was compared to a second "fast" calibration protocol, adapted from a previous method (J. H. Buffi et al. 2014), 
the mean precision errors from the across-subject calibration were approximately $3.3^{\circ}$ smaller than those from the fast calibration (Table 3). More specifically, mean precision errors of IP and MCP joints from the across-subject calibration were smaller than those from the fast calibration. Highest bias and precision errors using the across-subject calibration corresponded to PIP5 flexion $\left(-10.30^{\circ}\right)$ and MCP5 flexion $\left(13.45^{\circ}\right)$, respectively. Highest bias and precision errors using the fast calibration correspond to MCP5 abduction $\left(24.45^{\circ}\right)$ and $\mathrm{MCP} 3$ flexion $\left(22.46^{\circ}\right)$, respectively.

\section{Insert Table III here}

Phase 4. AROM obtained for the pathologic subject were in accordance with the rehabilitation assessment performed by clinicians. The AROM were out of the normal range for $\mathrm{CMC} 1$ extension and abduction, IP1 flexion and extension, DIP2 to DIP5 flexion and extension, and in particular for MCP2 to MCP4 flexion the AROM was out of the range of the calibration $\left(70^{\circ}, 60^{\circ}\right.$ and $59^{\circ}$ respectively, all of them lower than the $75^{\circ}$ required). These limitations would disable the subject for performing the static postures of the Detailed calibration protocol, and obviously the cycles needed to account for the crosscoupling effect.

\section{DISCUSSION}

The results of this work demonstrate promising approaches with strong potential to overcome critical problems associated with effective calibration of instrumented gloves. Such potential solutions are needed to advance technical capabilities for quantitative data collection during complex hand motions. First of all, we show that using a single, detailed calibration session for data collection from a single subject over multiple experimental 
sessions introduces only minimal error (mean precision error $3.50^{\circ}$ ), enabling data collection from the same subject over multiple days, without repeating a tedious, timeconsuming calibration procedure. Furthermore, we propose that the small errors associated with using our across-subject calibration protocol (mean precision error $4.45^{\circ}$ ) are acceptable for many purposes. In addition to the reduction in time and effort associated with glove calibration, in the scenarios in which the error levels are permissible, this approach also has the potential to improve the accuracy with which hand kinematics can be quantified when the subject has a severe disability that interferes with the capacity to achieve subsets of hand postures essential for completion of the detailed, individualized calibration. Specifically, in this case, the results of our study suggest that the across-subject calibration would give better results than a detailed calibration in which some sensors could not be properly calibrated.

When comparing the difference between the joint angles that resulted when the same sensor outputs were transformed with both the across-subject calibration and the detailed subjectspecific calibration, only three degrees of freedom (abduction of the little MCP joint and flexion and abduction of $\mathrm{CMC} 1$ joint) yielded errors greater than $10^{\circ}$. Due to the complexity of the base joint of the thumb and the location of the sensors, it is not surprising that errors associated with $\mathrm{CMC} 1$ were relatively large. The larger error associated with abduction of the little finger can be explained because it represents an accumulated error. Specifically, flexion of little MCP joint is calculated as the sum of the relative abduction between little and ring fingers and the relative abduction between ring and middle fingers, yielding an accumulated error of a magnitude of approximately twice the error of similar joints. 
The results of our correlation analysis suggest that, when using the across-subject calibration, several joint angles are sensitive to hand size. This result is consistent with the motivation of a previous study that used hand segment lengths as an input to ANN as an algorithm to transform sensor output to joint angles (Zhou, Malric, and Shirmohammadi 2010); although the success of this previous technique was not evaluated in terms of joint angle errors. We observed less sensitivity to hand breadth than hand length (e.g., only 4 degrees of freedom yielded significant correlations with hand breadth vs. 7 with hand length, Fig. 5). For MCP flexion, we note that 3 out of $5 \mathrm{MCP}$ joints were negatively correlated to either hand length or breadth. Thus, using the across-subject calibration instead of using the detailed calibration generally yielded greater MCP flexion angles for smaller hands and smaller MCP flexion angles for larger hands. Overall, our correlation analysis suggests that the degree of variability in hand sizes across a group of subjects should be considered when implementing the across-subject calibration approach, especially if the application requires data of high precision.

When a single subject adopted five static postures and the joint angles estimated using the across-subject calibration were compared to the photogrammetric technique, we observed a small, negative bias error across all joints (e.g., on average, the joint angles were smaller for the across-subject data). In contrast, we note a small, positive bias error for the fast calibration. While our interpretation is limited by the fact that the videogrammetric data had to be taken separately, we postulate that the result of a negative bias (e.g., smaller joint excursions from the neutral posture) is consistent with the fact that the individuals were wearing a glove, increasing joint stiffness. In addition, another source of bias in the across- 
subject approach is that abduction angles of fingers were obtained assuming no abduction for the middle finger, which may affect the recorded values for the other abduction angles.

Given the benefits of instrumented gloves for quantification of complex hand movements discussed previously, our analysis suggests that the across-subject calibration approach is a feasible methodology for many applications in which the measurement of joint angles is required (ranging from clinical diagnosis, rehabilitation or functional assessment, to robotics). Because we observed smaller differences relative to a reference data set (Table 3), we conclude the across-subject calibration methodology performed more effectively than the fast calibration protocol (grand mean precision errors $7.08^{\circ}$ and $10.31^{\circ}$, respectively). While this analysis was completed with an 18-sensor Cyberglove, it is extendable to a 22-sensor Cyberglove, which registers also fingers distal IP joint flexion. In order to use it, an analogous procedure to that presented for the rest of IP joints could be used.

\section{ACKNOWLEDGMENT}

We thank Marta Mora, $\mathrm{PhD}$, for her collaboration in coding for data glove acquisition, and the graduate student Sheyla Mestre Vicente for her collaboration in data collection.

This research was funded by the Universitat Jaume I through projects P1·1B2013-33 and P1- 1B2014-10, and by the Spanish Ministry of Economy and Competitiveness and the EU (FEDER funds) through project DPI2014-52095-P. This work was also funded by the NIH Grant NIH R01EB011615.

This article has no relevant Conflict of Interests. 


\section{NOMENCLATURE}

ADL

ANN

AROM

ASL

$\mathrm{CMC1}$

DIP2 to DIP5

DoF

HB

HL

IP

IP1

MCP

MCP1 to MCP5

PIP2 to PIP5

RMSE

SD
Activities of daily living

Artificial neural networks

Active range of motion

American sign language

Carpometacarpal joint of thumb

Distal interphalangeal joints (2 to 5, index to little digits)

Degree of freedom

Hand breadth

Hand length

Interphalangeal joint

Thumb interphalangeal joint

Metacarpophalangeal joint

Metacarpophalangeal joints ( 1 to 5 , thumb to little digits)

Proximal interphalangeal joints (2 to 5, index to little digits)

Root mean square error

Standard deviation 


\section{REFERENCES}

Brand, P W, and A M Hollister. 1999. Clinical Mechanics of the Hand. 3rd Editio. St. Louis: Mosby Publishing.

Buffi, James H, Joseph J Crisco, and Wendy M Murray. 2013. “A Method for Defining Carpometacarpal Joint Kinematics from Three-Dimensional Rotations of the Metacarpal Bones Captured in Vivo Using Computed Tomography." Journal of biomechanics 46(12): 2104-8.

Buffi, James H, Joaquín Luis Sancho Bru, Joseph J Crisco, and Wendy M Murray. 2014. "Evaluation of Hand Motion Capture Protocol Using Static Computed Tomography Images: Application to an Instrumented Glove." Journal of biomechanical engineering 136(12): 124501.

Buffi, James, Joaquín Luis Sancho Bru, Wendy M Murray, and Joseph J Crisco. 2014. "Evaluation of Hand Motion Capture Protocol Using Computed Tomography: Application to an Instrumented Glove." Journal of biomechanical engineering.

Bullock, Ian M., Joshua Z. Zheng, Sara De La Rosa, Charlotte Guertler, and Aaron M. Dollar. 2013. "Grasp Frequency and Usage in Daily Household and Machine Shop Tasks." IEEE Transactions on Haptics 6(3): 296-308.

Chiu, H Y, S C Lin, F C Su, S T Wang, and H Y Hsu. 2000. "The Use of the Motion Analysis System for Evaluation of Loss of Movement in the Finger." Journal of Hand Surgery (British and European Volume) 25(2): 195-99.

Crasborn, O., H. Sloetjes, E. Auer, and P. Wittenburg. 2006. "Combining Video and Numeric Data in the Analysis of Sign Languages with the ELAN Annotation Software." In 2nd Workshop on the Representation and Processing of Sign Languages: Lexicographic Matters and Didactic Scenarios, ed. Max Planck Institute for Psycholinguistics. Paris: ELRA, 82-87.

Eccarius, Petra, Rebecca Bour, and Robert A Scheidt. 2012. "Dataglove Measurement of Joint Angles in Sign Language Handshapes." Sign language and linguistics 15(1): 3972.

Griffin, Weston B, Ryan P Findley, Michael L Turner, and Mark R Cutkosky. 2000. "Calibration and Mapping of a Human Hand for Dexterous Telemanipulation." In ASME IMECE 2000 Symposium on Haptic Interfaces for Virtual Environments and Teleoperator Systems, 1-8.

Grinyagin, I V, E V Biryukova, and M A Maier. 2005. "Kinematic and Dynamic Synergies of Human Precision-Grip Movements." Journal of neurophysiology 94: 2284-94.

Holzbaur, Katherine R S, Wendy M Murray, and Scott L Delp. 2005. "A Model of the Upper Extremity for Simulating Musculoskeletal Surgery and Analyzing Neuromuscular Control." Annals of biomedical engineering 33(6): 829-40.

Kessler, G. Drew, Larry F. Hodges, and Neff Walker. 1995. "Evaluation of the CyberGlove as a Whole-Hand Input Device." ACM Transactions on Computer-Human Interaction 2(4): 263-83.

Kortier, Henk G, Victor I Sluiter, Daniel Roetenberg, and Peter H Veltink. 2014. 
“Assessment of Hand Kinematics Using Inertial and Magnetic Sensors.” Journal of neuroengineering and rehabilitation 11(1): 70.

Kramer, James F. 1996. "Determination of Thumb Position Using Measurements of Abduction and Rotation.".

Metcalf, Cheryl D, Rebecca Robinson, Adam J Malpass, Tristan P Bogle, Thomas A Dell, Chris Harris, and Sara H Demain. 2013. "Markerless Motion Capture and Measurement of Hand Kinematics: Validation and Application to Home-Based Upper Limb Rehabilitation." IEEE transactions on bio-medical engineering 60(8): 2184-92.

Mitobe, Kazutaka, Takaaki Kaiga, Takashi Yukawa, Takeshi Miura, Hideo Tamamoto, Al Rodgers, and Noboru Yoshimura. 2006. "Development of a Motion Capture System for a Hand Using a Magnetic Three Dimensional Position Sensor.” In ACM SIGGRAPH 2006 Research Posters on - SIGGRAPH '06, New York, New York, USA: ACM Press, 102.

Nathan, Dominic E., Michelle J. Johnson, and John R. McGuire. 2009. "Design and Validation of Low-Cost Assistive Glove for Hand Assessment and Therapy during Activity of Daily Living-Focused Robotic Stroke Therapy." The Journal of Rehabilitation Research and Development 46(5): 587.

O ’flynn, Brendan, Javier Torres Sanchez, James Connolly, Joan Condell, Kevin Curran, Philip Gardiner, Barry Downes, and Barry Downes Tssg. 2015. "Integrated Smart Glove for Hand Motion Monitoring." SENSORDEVICES 2015 The 6th International Conference on Sensor Device Technologies and Applications, Venize, Italy, Aug 2328 '15

Oess, Ninja P, Johann Wanek, and Armin Curt. 2012. "Design and Evaluation of a LowCost Instrumented Glove for Hand Function Assessment." Journal of neuroengineering and rehabilitation 9: 2.

Sanchez-Margallo, F.M., J.A. Sanchez -Margallo, J.B. Pagador, J.L. Moyano, J. Moreno, and J. Uson. 2010. "Ergonomic Assessment of Hand Movements in Laparoscopic Surgery Using the Cyberglove (R)." In Computational Biomechanics for Medicine, eds. Karol Miller and Poul M.F. Nielsen. New York, NY: Springer New York, 12128 .

Sancho-Bru, Joaquin L, Nestor J Jarque-Bou, Margarita Vergara, and Antonio PerezGonzalez. 2014. "Validity of a Simple Videogrammetric Method to Measure the Movement of All Hand Segments for Clinical Purposes." Proceedings of the Institution of Mechanical Engineers. Part H, Journal of engineering in medicine.

Sancho-Bru, Joaquín L., Marta C. Mora, Beatriz E. León, Antonio Pérez-González, José L. Iserte, and Antonio Morales. 2012. "Grasp Modelling with a Biomechanical Model of the Hand." Computer methods in biomechanics and biomedical engineering 17(4): 297-310.

Tripp, Brady L, Tim L Uhl, Carl G Mattacola, Cidambi Srinivasan, and Robert Shapiro. 2006. "A Comparison of Individual Joint Contributions to Multijoint Position Reproduction Acuity in Overhead-Throwing Athletes." Clinical biomechanics (Bristol, Avon) 21(5): 466-73. 
Vergara, Margarita, J L Sancho-Bru, V Gracia-Ibáñez, and A Pérez-González. 2014. “An Introductory Study of Common Grasps Used by Adults during Performance of Activities of Daily Living." Journal of hand therapy: official journal of the American Society of Hand Therapists.

Zheng, Joshua Z., Sara De La Rosa, and Aaron M. Dollar. 2011. “An Investigation of Grasp Type and Frequency in Daily Household and Machine Shop Tasks." In 2011 IEEE International Conference on Robotics and Automation (ICRA), Affiliation: Department of Mechanical Engineering and Materials Science, School of Engineering and Applied Science, Yale University, New Haven, CT, United States;

Correspondence Address: Zheng, J.Z.; Department of Mechanical Engineering and Materials Scie, 4169-75.

Zhou, Jilin Zhou Jilin, François Malric, and Shervin Shirmohammadi. 2010. "A New HandMeasurement Method to Simplify Calibration in Cyberglove-Based Virtual Rehabilitation." IEEE Transactions on Instrumentation and Measurement 59: 24962504. 


\section{Figure Captions List}

Fig. 1 18-sensor Cyberglove sensor location (the two sensors related to the wrist are not sketched as they are not used)

Fig. 2 Postures and guided movements used for the calibration protocol: (a) Examples of calibration trials 1 to 20; (b) Trials 21 and 22; (c) Starting postures of trials 23 to 25; (d) Examples of trials 23 to 40; (e) Neutral extension and abduction for trials 41 and 42; (f) Maximal extension during trial 41; (g) Maximal abduction (left) and adduction (right) during trial 42; (h) closed loop movement during trial 43; (i) Measurement of little CMC flexion in trials 21 (left) and 44 (right)

Fig. 3 Static postures used to evaluate the errors of the method proposed: (a) Maximal abduction of all fingers with hand in a plane; (b) All fingers in $90^{\circ}$ MCP flexion while thumb in maximal extension; (c) Grasping a ball; (d) Letter 'Y' from American Sign Language (ASL); (e) Letter 'R' from ASL

Fig. 4 Right hand of the injured subject.

Fig. 5 Visual comparison of the five static postures for the subject 9, the one with the highest differences (detailed subject-specific calibration versus across-subject calibration). (a) Maximal abduction of all fingers; (b) All fingers in $90^{\circ} \mathrm{MCP}$ flexion while thumb in maximal extension; (c) Grasping a ball; (d) Letter ' $Y$ ' from American Sign Language (ASL); (e) Letter 'R' from ASL

Fig. 6 Pearson correlations of the precision errors with hand breadth (HB) and hand length (HL): darker shadowed cells for $\mathrm{p}<0.01$, lighter shadowed cells for $\mathrm{p}<$ 0.05. The sign of the correlations is also shown 


\section{Table Caption List}

Table 1 Descriptive data of all the subjects participating in the experiments

Table 2 Mean bias and precision errors, in degrees, of using gains and coefficients obtained with the detailed calibration in a different session for subjects of sample 1 (phase 1), and of using the across-subject calibration versus the detailed subject-specific calibration (phase 2)

Table 3 Mean values for bias and precision errors of using the across-subject calibration and the subject-specific fast calibration compared to using the videogrammetric technique 


\section{FIGURES}

Fig. 1

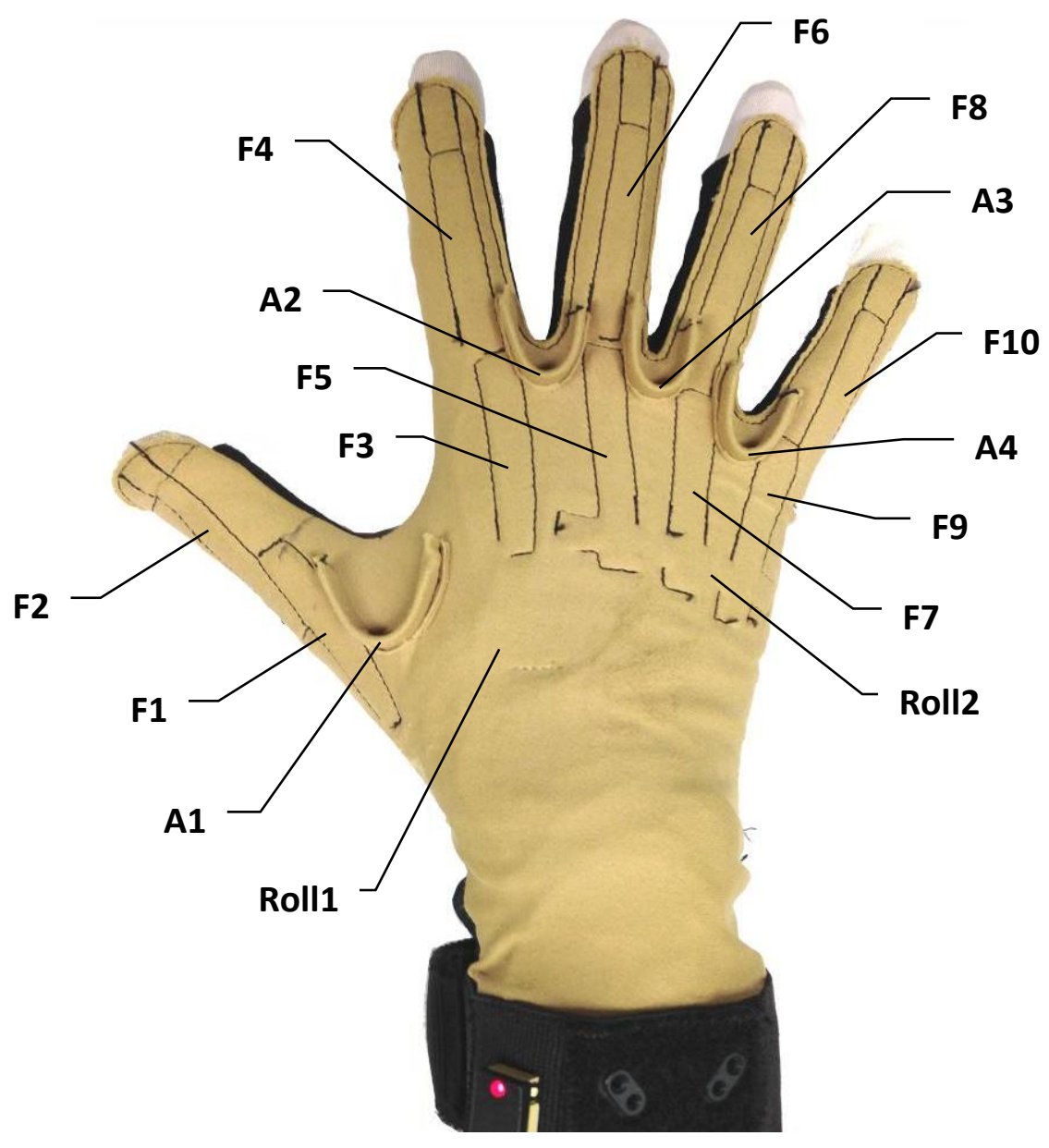


Fig. 2
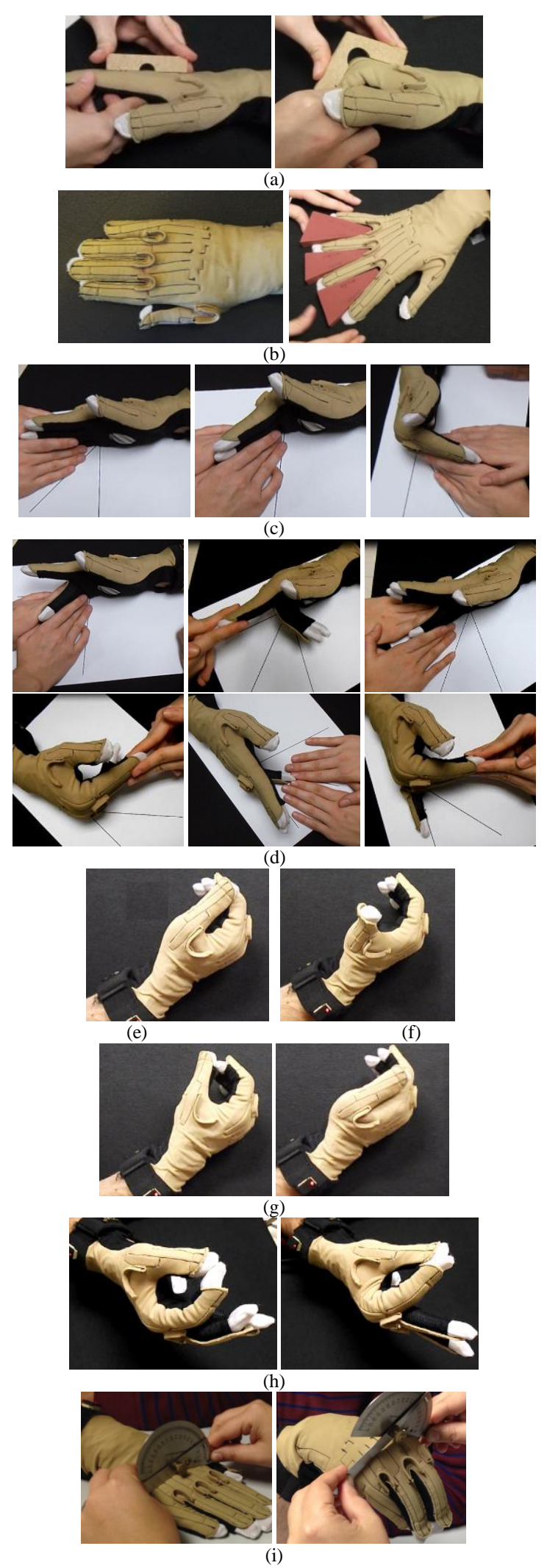
Fig. 3

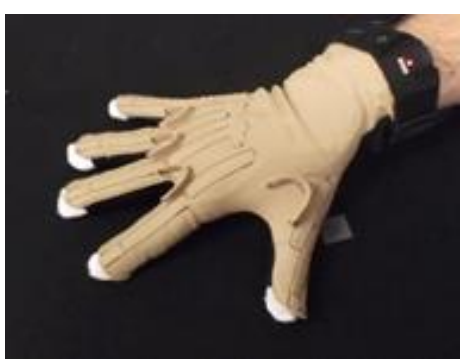

(a)

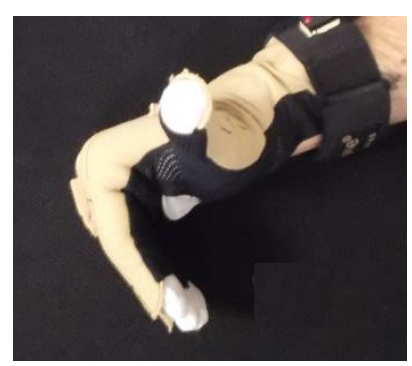

(b)

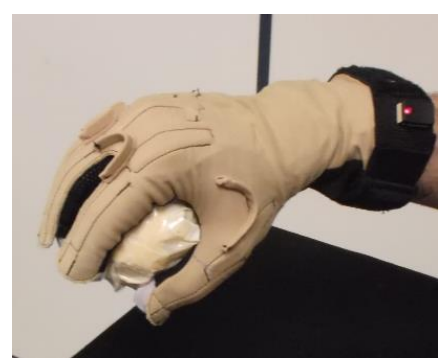

(c)

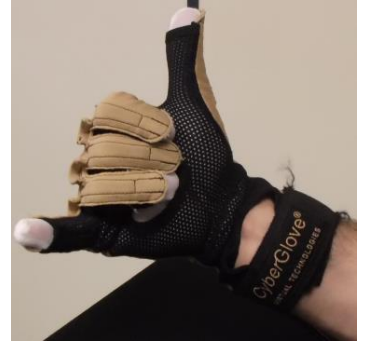

(d)

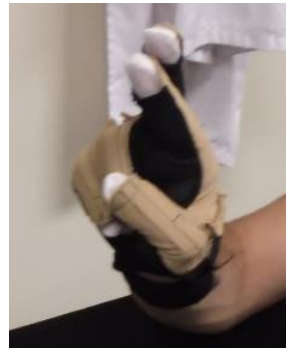

(e) 
Fig. 4

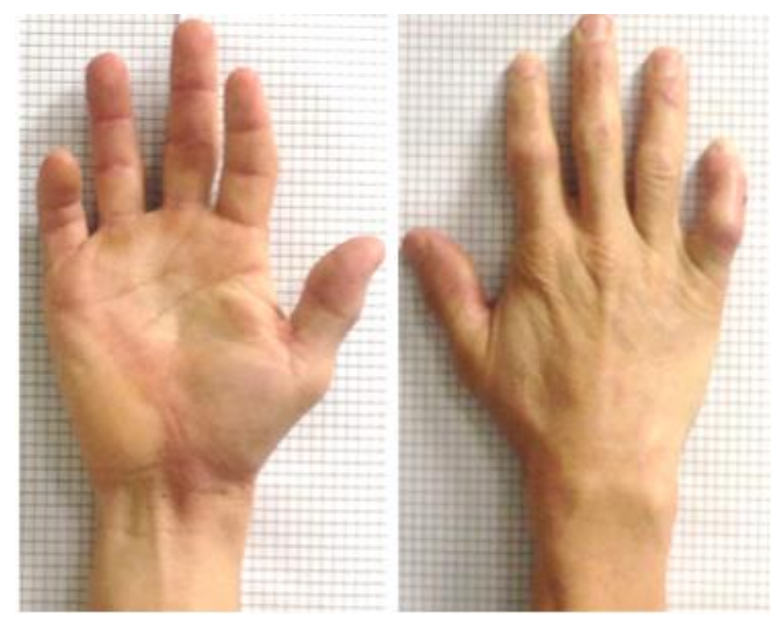


Fig. 5

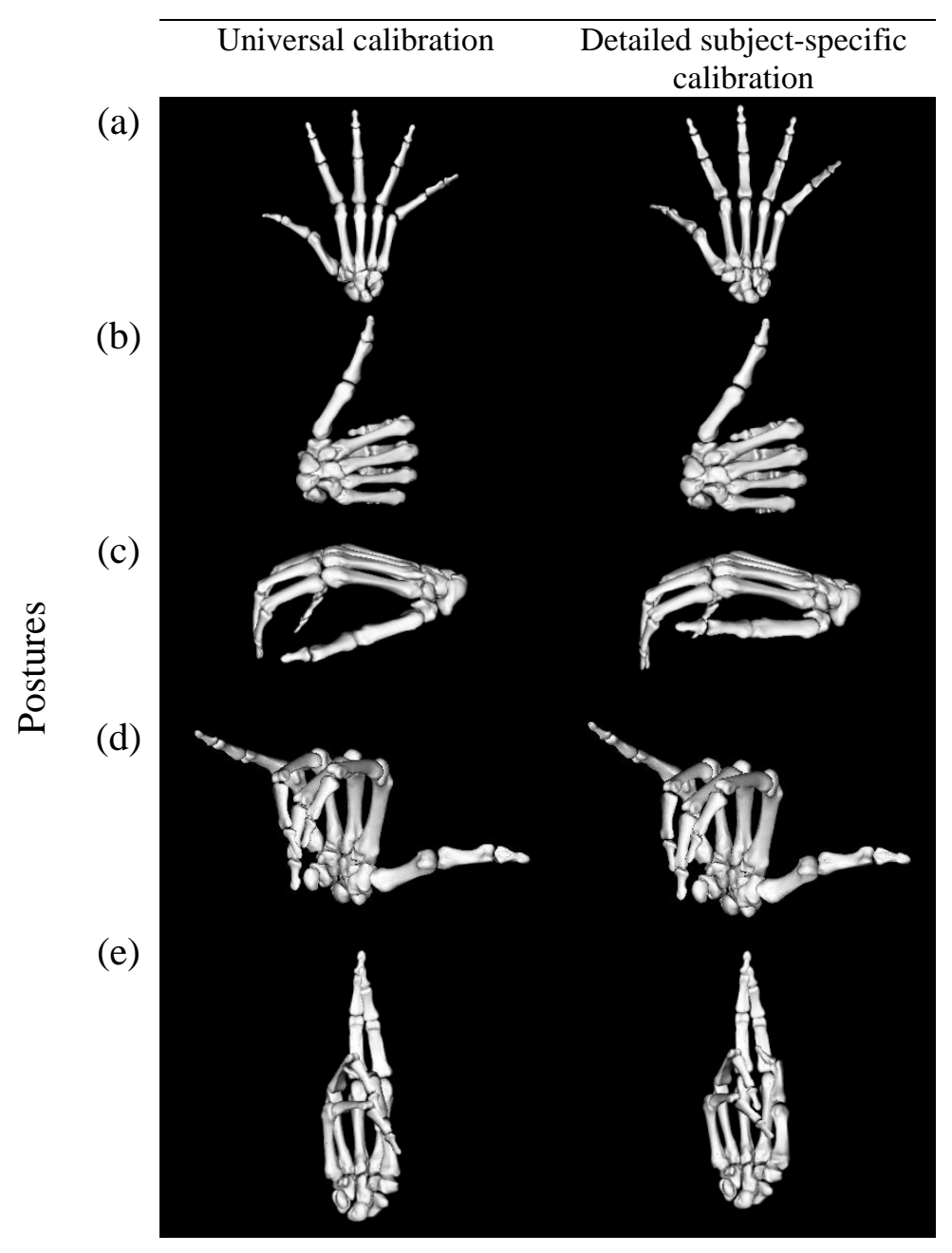


Fig. 6

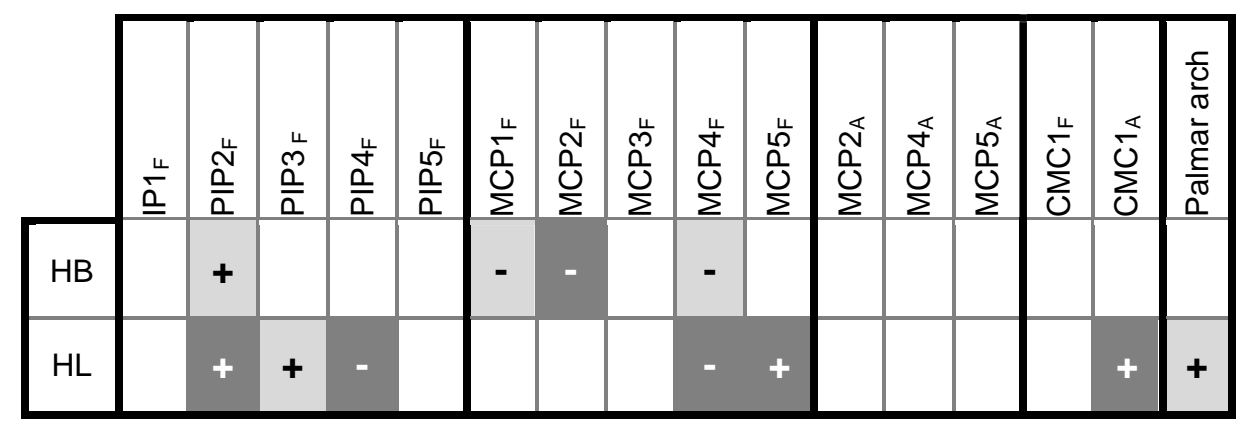




\section{TABLES}

Table I

\begin{tabular}{llccc}
\hline & & Gender & HL $(\mathrm{mm})^{\mathrm{a}}$ & HB $(\mathrm{mm})^{\mathrm{b}}$ \\
\hline Sample 1 & Subject 1 & Male & 183 & 88 \\
& Subject 2 & Female & 169 & 76 \\
& Subject 3 & Male & 176 & 81 \\
& Subject 4 & Female & 160 & 69 \\
& Subject 5 & Male & 196 & 83 \\
& Subject 6 & Female & 179 & 76 \\
\hline Sample 2 & Subject 7 & Male & 171 & 86 \\
& Subject 8 & Female & 166 & 73 \\
& Subject 9 & Male & 204 & 82 \\
& Subject 10 & Female & 176 & 74 \\
\hline
\end{tabular}

${ }^{\mathrm{a}} \mathrm{HL}$ : hand length, measured from the proximal palmar crease to the tip of the middle finger)

${ }^{\mathrm{b}} \mathrm{HB}$ : hand breath, measured at the metacarpal heads 
Table II

\begin{tabular}{|c|c|c|c|c|c|c|}
\hline \multicolumn{4}{|c|}{ Phase 1} & \multicolumn{3}{|c|}{ Phase 2} \\
\hline Joint & $\operatorname{Bias}\left({ }^{\circ}\right)$ & $\begin{array}{c}\text { Precision } \\
\left({ }^{\circ}\right)\end{array}$ & $\begin{array}{l}\text { Mean Precision of } \\
\text { group of joints }\left(^{\circ}\right)\end{array}$ & $\operatorname{Bias}\left({ }^{\circ}\right)$ & $\begin{array}{c}\text { Precision } \\
\left({ }^{\circ}\right)\end{array}$ & $\begin{array}{l}\text { Mean Precision of } \\
\text { group of joints }\left({ }^{\circ}\right)\end{array}$ \\
\hline $\mathrm{IP} 1_{\mathrm{f}}$ & 0.01 & 1.33 & & -0.03 & 1.72 & \\
\hline $\mathrm{PIP} 2_{\mathrm{f}}$ & -0.02 & 2.88 & & 0.04 & 2.47 & \\
\hline $\mathrm{PIP}_{\mathrm{f}}$ & 0.02 & 3.76 & 2.71 & -0.41 & 1.34 & 1.74 \\
\hline $\mathrm{PIP}_{\mathrm{f}}$ & 0.01 & 4.00 & & 0.72 & 1.70 & \\
\hline $\mathrm{PIP5}_{\mathrm{f}}$ & 1.32 & 1.59 & & -0.35 & 1.48 & \\
\hline $\mathrm{MCP}_{1}$ & 0.00 & 0.92 & & 0.84 & 4.90 & \\
\hline $\mathrm{MCP} 2_{\mathrm{f}}$ & -0.05 & 2.21 & & -0.23 & 2.10 & \\
\hline $\mathrm{MCP}_{\mathrm{f}}$ & 0.00 & 2.15 & 1.62 & 2.26 & 3.00 & 2.67 \\
\hline $\mathrm{MCP}_{\mathrm{f}}$ & -0.01 & 1.76 & & 0.19 & 1.44 & \\
\hline $\mathrm{MCP}_{\mathrm{f}}$ & 0.92 & 1.04 & & -1.51 & 1.91 & \\
\hline $\mathrm{MCP} 2_{\mathrm{a}}$ & -0.25 & 4.77 & & 0.2 & 5.34 & \\
\hline $\mathrm{MCP}_{\mathrm{a}}$ & 0.00 & 3.31 & 3.52 & -0.4 & 8.19 & 7.90 \\
\hline $\mathrm{MCP}_{\mathrm{a}}$ & 3.19 & 2.47 & & 4.86 & 10.16 & \\
\hline $\mathrm{CMC1}_{\mathrm{f}}$ & -1.26 & 12.66 & & 1.78 & 10.26 & \\
\hline CMC1 & 0.08 & 2.88 & & 2.18 & 10.39 & \\
\hline $\begin{array}{l}\text { Palmar } \\
\text { arch }\end{array}$ & 1.79 & 8.19 & & -0.18 & 4.85 & \\
\hline $\begin{array}{l}\text { Grand } \\
\text { Mean }\end{array}$ & 0.36 & 3.50 & & $\overline{0.49}$ & 4.45 & \\
\hline
\end{tabular}


Table III

\begin{tabular}{|c|c|c|c|c|c|c|}
\hline \multirow[b]{2}{*}{ Joint } & \multicolumn{3}{|c|}{ Across-subject calibration } & \multicolumn{3}{|c|}{ Subject-specific fast calibration } \\
\hline & $\operatorname{Bias}\left({ }^{\circ}\right)$ & $\begin{array}{c}\text { Precision } \\
\left({ }^{\circ}\right)\end{array}$ & $\begin{array}{l}\text { Mean Precision of } \\
\text { group of joints }\left(^{\circ}\right)\end{array}$ & $\operatorname{Bias}\left({ }^{\circ}\right)$ & $\begin{array}{c}\text { Precision } \\
\left({ }^{\circ}\right)\end{array}$ & $\begin{array}{l}\text { Mean Precision of } \\
\text { group of joints }\left({ }^{\circ}\right)\end{array}$ \\
\hline$\overline{\mathrm{IP}} 1_{\mathrm{f}}$ & -0.65 & 1.50 & & -2.20 & 9.13 & \\
\hline $\mathrm{PIP} 2_{\mathrm{f}}$ & -3.09 & 5.56 & & -12.51 & 11.25 & \\
\hline $\mathrm{PIP} 3_{\mathrm{f}}$ & -4.86 & 9.40 & 6.26 & -4.56 & 9.25 & 9.13 \\
\hline $\mathrm{PIP}_{\mathrm{f}}$ & -3.77 & 4.68 & & -5.19 & 5.87 & \\
\hline $\mathrm{PIP}_{\mathrm{f}}$ & -10.30 & 10.19 & & -9.80 & 10.12 & \\
\hline$\overline{\mathrm{MCP}} 1_{\mathrm{f}}$ & 5.62 & 6.00 & & -4.66 & 2.20 & \\
\hline $\mathrm{MCP} 2_{\mathrm{f}}$ & -2.28 & 6.64 & & 8.31 & 13.20 & \\
\hline $\mathrm{MCP}_{\mathrm{f}}$ & -0.60 & 8.20 & 7.29 & 14.78 & 22.46 & 13.32 \\
\hline $\mathrm{MCP}_{\mathrm{f}}$ & -9.87 & 2.17 & & 5.34 & 16.93 & \\
\hline $\mathrm{MCP}_{\mathrm{f}}$ & 7.92 & 13.45 & & 3.95 & 11.83 & \\
\hline$\overline{\mathrm{MCP}} 2_{\mathrm{a}}$ & 1.76 & 7.26 & & 1.19 & 10.29 & \\
\hline $\mathrm{MCP}_{\mathrm{a}}$ & -0.05 & 8.18 & 8.08 & 5.68 & 10.00 & 11.47 \\
\hline $\mathrm{MCP}_{\mathrm{a}}$ & 4.01 & 8.80 & & 24.45 & 14.11 & \\
\hline$\overline{\mathrm{CMC}} 1_{\mathrm{f}}$ & -3.63 & 5.76 & & 6.62 & 7.43 & \\
\hline $\mathrm{CMC1}_{\mathrm{a}}$ & -1.73 & 6.81 & & -4.70 & 3.18 & \\
\hline $\begin{array}{l}\text { Palmar } \\
\text { arch }\end{array}$ & -0.41 & 8.69 & & -2.71 & 7.66 & \\
\hline $\begin{array}{l}\text { Grand } \\
\text { Mean }\end{array}$ & -1.37 & 7.08 & & 1.50 & 10.31 & \\
\hline
\end{tabular}

